\title{
VECSEL-based frequency comb in the MIR
}

Robert Rockmore, Alexandre Laurain, Jerome V. Moloney, R. Jason Jones

Robert Rockmore, Alexandre Laurain, Jerome V. Moloney, R. Jason Jones, "VECSEL-based frequency comb in the MIR," Proc. SPIE 10901, Vertical External Cavity Surface Emitting Lasers (VECSELs) IX, 1090108 (4 March 2019); doi: 10.1117/12.2510657

SPIE. Event: SPIE LASE, 2019, San Francisco, California, United States 


\title{
VECSEL-based frequency comb in the MIR
}

\author{
Robert Rockmore, Alexandre Laurain, Jerome V. Moloney, R. Jason Jones \\ College of Optical Sciences, The University of Arizona, 1630 E University Blvd, Tucson, AZ, \\ US 85719;
}

\begin{abstract}
The mid-infrared (MIR) region above 3 microns is of great interest for spectroscopic applications. Because it is difficult to produce modelocked laser sources that emit natively in this region, difference frequency generation (DFG) is a popular method to produce mid-IR output using more traditional laser oscillators. Previous examples include fiber based DFG sources and OPOs, which are typically limited to repetition rates on the order of tens to hundreds of MHz. VECSELs allow access to higher repetition rates, while the use of highly nonlinear waveguides enables the requisite spectral broadening despite the lower pulse energy.

In this work we present a VECSEL-based frequency comb that uses DFG to produce output in the 3-4 micron range. This system is based on a modelocked VECSEL emitting at a $1030 \mathrm{~nm}$ wavelength with a 1.6 $\mathrm{GHz}$ repetition rate. A $\mathrm{Yb}$ fiber amplification system is used to increase the power to over $10 \mathrm{~W}$ and compress the pulses to sub-90 fs. Coherent spectral broadening out to $1560 \mathrm{~nm}$ is achieved with a nonlinear waveguide. By combining the $1030 \mathrm{~nm}$ and $1560 \mathrm{~nm}$ beams in a PPLN DFG crystal, $290 \mathrm{~mW}$ of mid IR output between 3.0 and 3.5 microns is produced. Since the DFG light is produced by two wavelengths from the same oscillator, the carrier envelope offset frequency is cancelled, producing an offset free comb requiring stabilization of only a single degree of freedom. We characterize this VECSEL based frequency comb and discuss the advantages it provides for spectroscopic applications.
\end{abstract}

Keywords: VECSELs, frequency combs, nonlinear broadening, supercontinuum generation, mid-infrared

\section{INTRODUCTION}

The MIR (2-20 $\mu \mathrm{m})$ contains the so-called "fingerprint region" where many molecules possess strong absorption features. This wavelength range has many important applications for spectroscopy and sensing, such as the detection of airborne pollutants and and industrial gasses, as well as detection of biological and chemical weapons. Optical frequency combs provide high resolution spectroscopy capabilities, but there are a limited number of such sources in the MIR region, such as quantum cascade lasers (QCLs), ${ }^{1}$ optical parametric oscillators (OPOs), ${ }^{2}$ and sources based on supercontinuum generation ${ }^{3}$ or difference frequency generation (DFG). ${ }^{4}$ QCLs are currently somewhat limited in wavelength coverage and power, while OPOs can produce broadly tunable output with powers of over $1 \mathrm{~W} .{ }^{5}$ However, they rely on complex cavities that must be actively locked. Supercontinuum generation can produce very broad spectra spanning from the near-IR (NIR) to the MIR, but the extreme broadening spreads the power out over a large wavelength range, which is not always desired. DFG based comb sources can access a large range of wavelengths and rely on more mature NIR modelocked laser sources. DFG sources that utilize coherent pump and signal beams from the same oscillator will additionally have a carrier envelope offset frequency $\left(f_{\text {ceo }}\right)$ that is fixed at zero, so that only the repetition rate $\left(f_{\text {rep }}\right)$ needs to be stabilized in order to fully stabilize the resulting optical comb. ${ }^{6}$ However, this requires a sufficiently broad and coherent spectrum in the NIR to reach the desired MIR frequency. This can be achieved either through intra-pulse dfg from very short pulses, ${ }^{7}$ or from nonlinear broadening and subsequent difference frequency generation. Most of the DFG combs demonstrated thus far were based on modelocked fiber ${ }^{8}$ and solid state lasers, which typically have repetition rates (and therefore comb line spacing) on the order of a few hundred $\mathrm{MHz}$ and have been used successfuly in dual comb configurations for spectroscopy. ${ }^{9}$

Modelocked vertical external cavity surface emitting lasers (VECSELs) can readily produce frequency combs in the NIR and offer good wavelength tunability and the potential for electrical pumping. While fiber and solid

Further author information:(Send correspondence to R. Rockmore)

R. Rockmore: E-mail: Rockmore@optics.arizona.edu

Vertical External Cavity Surface Emitting Lasers (VECSELs) IX, edited by

Ursula Keller, Proc. of SPIE Vol. 10901, 1090108 - @ 2019 SPIE

CCC code: $0277-786 \mathrm{X} / 19 / \$ 18 \cdot$ doi: $10.1117 / 12.2510657$

Proc. of SPIE Vol. 10901 1090108-1 
state lasers often suffer from $Q$ switching instabilities at higher repetition rates, ${ }^{10,11}$ VECSELs have demonstrated repetition rates as high as $100 \mathrm{GHz}^{12}$ and pulse durations below $100 \mathrm{fs}^{13,14}$ with stable, fundamental modelocking. Frequency combs with repetition rates from 1-3 GHz are of special interest for spectroscopy applications, especially for complex molecules with large $(>10 \mathrm{GHz})$ linewidths absorbing in the MIR. These higher repetition rates give a higher power per comb line for a given average power, more easily resolved comb lines ${ }^{15}$ and higher acquisition rates for dual comb spectroscopy while still providing sufficient resolution to detect the molecules of interest. However, most femtosecond laser oscillators produce pulses with at most tens of $\mathrm{nm}$ of spectral bandwidth, which is insufficient to reach the MIR region through difference frequency generation. As a result, a nonlinear medium must be used to externally spectrally broaden the output to the hundreds of $\mathrm{nm}$ of wavelength span necessary. For $\mathrm{MHz}$ repetition rate sources this is typically achieved with highly nonlinear fibers, ${ }^{16}$ but for repetition rates above $1 \mathrm{GHz}$ and lower pulse energies these media are often not sufficient to produce the desired broadening. Chip based nonlinear waveguides provide higher nonlinearity, broad transparency, and can be designed for a desired dispersion and output spectrum, making them ideal for the coherent broadening that high repetition rate DFG combs require. ${ }^{17}$ By utilizing a modelocked VECSEL oscillator and additional fiber amplification along with a $S_{3} N_{4}$ (silicon nitride, or SiN) waveguide, we have developed a MIR frequency comb based on DFG with coherent output spanning from 3-3.5 $\mu \mathrm{m}$ and a $1.61 \mathrm{GHz}$ repetition rate. DFG is achieved using $\mathrm{MgO}$ doped periodically poled lithium niobate. which is readily available and relatively efficient at wavelengths below $5 \mu \mathrm{m}$. For longer wavelength DFG, orientation-patterned gallium phosphide is often used. ${ }^{7}$

\section{EXPERIMENTAL SETUP}

The system is based on a passively modelocked VECSEL with an emission wavelength of $1030 \mathrm{~nm}$. The overall system layout is shown in figure 1 . The VECSEL is configured in a v-cavity geometry, with a flat semiconductor saturable absorber mirror (SESAM) and $0.6 \%$ output coupling mirror with $10 \mathrm{~cm}$ radius of curvature forming the end mirrors. The cavity also contains an additional highly reflective folding mirror between the output coupler and gain chip. This mirror is mounted to a piezoelectric transducer (PZT) to allow for cavity length control and active repetition rate stabilization. The gain structure used in this laser consists of a hybrid metalsemiconductor distributed Bragg reflector and an active region made up of 12 non uniformly arranged quantum wells for gain under 4 antinodes of the electric field. The composition and design of the gain and SESAM chips have been described previously and has been used to produce sub 100 fs pulses. ${ }^{14}$ The chip was coated with a $\mathrm{Ta}_{2} \mathrm{O}_{5} / \mathrm{SiO}_{2}$ bilayer antireflective coating to obtain a near zero dispersion at the lasing wavelength. The VECSEL oscillator was designed for stable fundamental modelocking operation at $1.61 \mathrm{GHz}$ and was placed in a rigid acrylic enclosure to isolate it from external noise sources and maximize free-running frequency stability. The VECSEL produces pulses approximately $1 \mathrm{ps}$ in duration with $130 \mathrm{~mW}$ of average power. The lasing spectrum and RF spectrum of the VECSEL is shown in figure 2, along with the spectrum at $1560 \mathrm{~nm}$ after the nonlinear broadening and amplification described in the following section.

In order to increase average power and decrease pulse duration, a Yb amplifier was used along with two grating pairs for pulse compression, as shown in 1. The first Yb amplifier stage is a preamplifier consisting of $2.5 \mathrm{~m}$ of double-clad polarization maintaining Yb doped fiber (Nufern PLMA-YDF-10/125) pumped by a $915 \mathrm{~nm}$ fiber coupled diode laser. The preamplifier increases the average power to $300 \mathrm{~mW}$ and is used to ensure that the second amplifier stage is adequately seeded to minimize amplified spontaneous emission and instabilities. After the preamplifier stage is a pair of transmission gratings in a double pass configuration for optimization of the chirp of the pulse launched into the second stage of the $\mathrm{Yb}$ amplifier. This stage consists of a $7 \mathrm{~m}$ length of the same gain fiber pumped by a pair of $915 \mathrm{~nm}$ fiber coupled diode lasers. The pulse undergoes nonlinear broadening inside the high power amplifier stage, resulting in the spectrum shown in figure 2. A second pair of gratings compresses the pulse to $87 \mathrm{fs}$ in duration at an average power of over $11 \mathrm{~W}$. The amplifier system was designed to manage dispersion such that the pulses can be cleanly compressed after broadening as described in previous work. ${ }^{18-20}$

After amplification and compression, the $1030 \mathrm{~nm}$ beam is split into two arms. One beam consists of $1 \mathrm{~W}$ of power that is coupled into a SiN waveguide for nonlinear broadening. Coupling is achieved with a .65 NA fused silica objective lens. The SiN waveguide is similar to that described in $\operatorname{ref}^{21}$ and is $900 \mathrm{~nm}$ in width 




Figure 1. Experimental setup. Output of $1030 \mathrm{~nm}$ VECSEL oscillator is amplified with a two stage Yb amplifier that features a pre-chirp grating after the first stage and pulse compression gratings after the second stage. One beam is coupled into a SiN waveguide and is broadened to $1560 \mathrm{~nm}$ where it is further amplified. $6 \mathrm{~W}$ of $1030 \mathrm{~nm}$ light and $600 \mathrm{~mW}$ of $1560 \mathrm{~nm}$ light are focused into a MgO:PPLN crystal to generate output from 3.0-3.5 $\mu \mathrm{m}$. A delay stage is used to spatially overlap the two pulses (not shown).
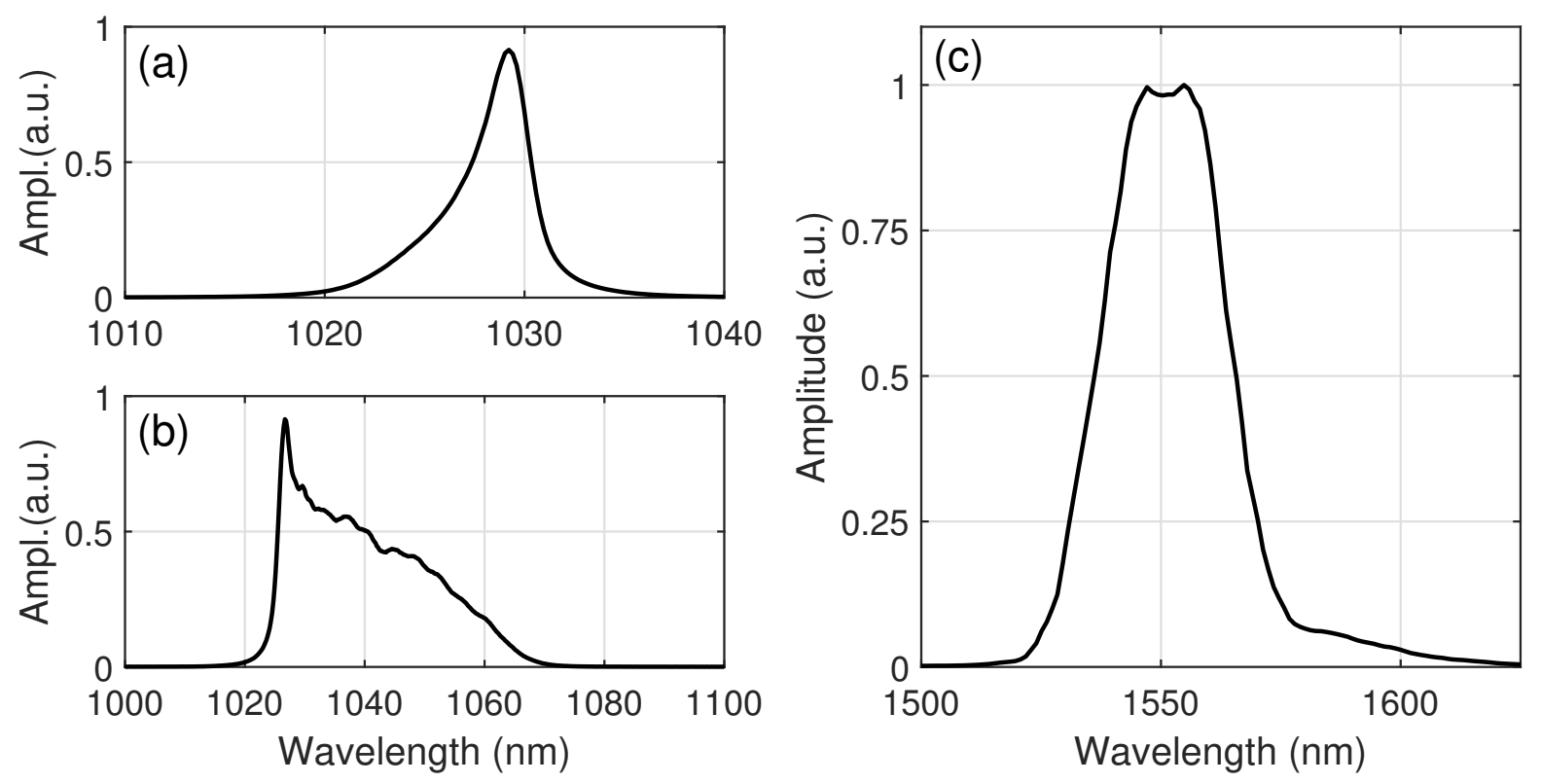

Figure 2. (a) Spectrum directly from modelocked VECSEL. (b) Spectrum after nonlinear amplification and broadening in $\mathrm{Yb}$ doped fiber amplifier. The amplified spectrum acts as the pump for the DFG process. (c) Spectrum after broadening to $1560 \mathrm{~nm}$ in SiN waveguide and amplification with Er amplifier. This spectrum acts as the signal for the DFG process that produces the MIR idler output. 
and $700 \mathrm{~nm}$ in height, with a total length of $20 \mathrm{~mm}$. Both ends of the waveguide feature inverse tapers to optimize coupling efficiency. This produces a spectrum that spans from $600 \mathrm{~nm}$ to beyond $1700 \mathrm{~nm}$, with a long wavelength peak extending to $1570 \mathrm{~nm}$. Approximately $3 \mathrm{~mW}$ of optical power is produced from $1530-1570 \mathrm{~nm}$. The supercontinuum spectrum exhibits this long wavelength peak at powers on the order of a few hundred $\mathrm{mW}$, as shown in figure 3. A higher power of $1 \mathrm{~W}$ was used to maximize the output around 1530-1570 nm without damaging the waveguide or reducing coherence. In order to increase the power in this wavelength range further, the output of the waveguide was coupled into a two stage amplifier designed for this wavelength band after first being collimated with a $.68 \mathrm{NA}$ aspheric lens. This amplifier consists of a $1.5 \mathrm{~m}$ length of single mode $\mathrm{Er}$ doped gain fiber (non polarization maintaining) followed by a linear polarizer and $2 \mathrm{~m}$ of double clad polarization maintaining Er/Yb co-doped gain fiber (Nufern PM-EYDF-12/130-HE). Spectral filtering of the input was achieved by exploiting the chromatic aberration of the asphere such that only the portion of the beam around $1560 \mathrm{~nm}$ was collimated and filtering the unwanted wavelengths with apertures. A fiber isolator was spliced to the input of the first amplifier stage to prevent back-reflections from the waveguide output facet from coupling back into the amplifier. A length of dispersion compensating fiber (DCF-38, $-38 \mathrm{ps} / \mathrm{nm} * \mathrm{~km}$ ) was spliced between the two stages to compress the pulse after the second amplifier stage to approximately $200 \mathrm{fs}$. The optical power after the second amplifier stage is $600 \mathrm{~mW}$ under typical operating conditions. The amplifier output was collimated before a delay stage to overlap the $1560 \mathrm{~nm}$ and $1030 \mathrm{~nm}$ pulses in space, and the collimating lens was chosen to optimize mode matching between the two beams. A half wave plate was used to match the polarization of the long wavelength beam to that of the $1030 \mathrm{~nm}$ beam.

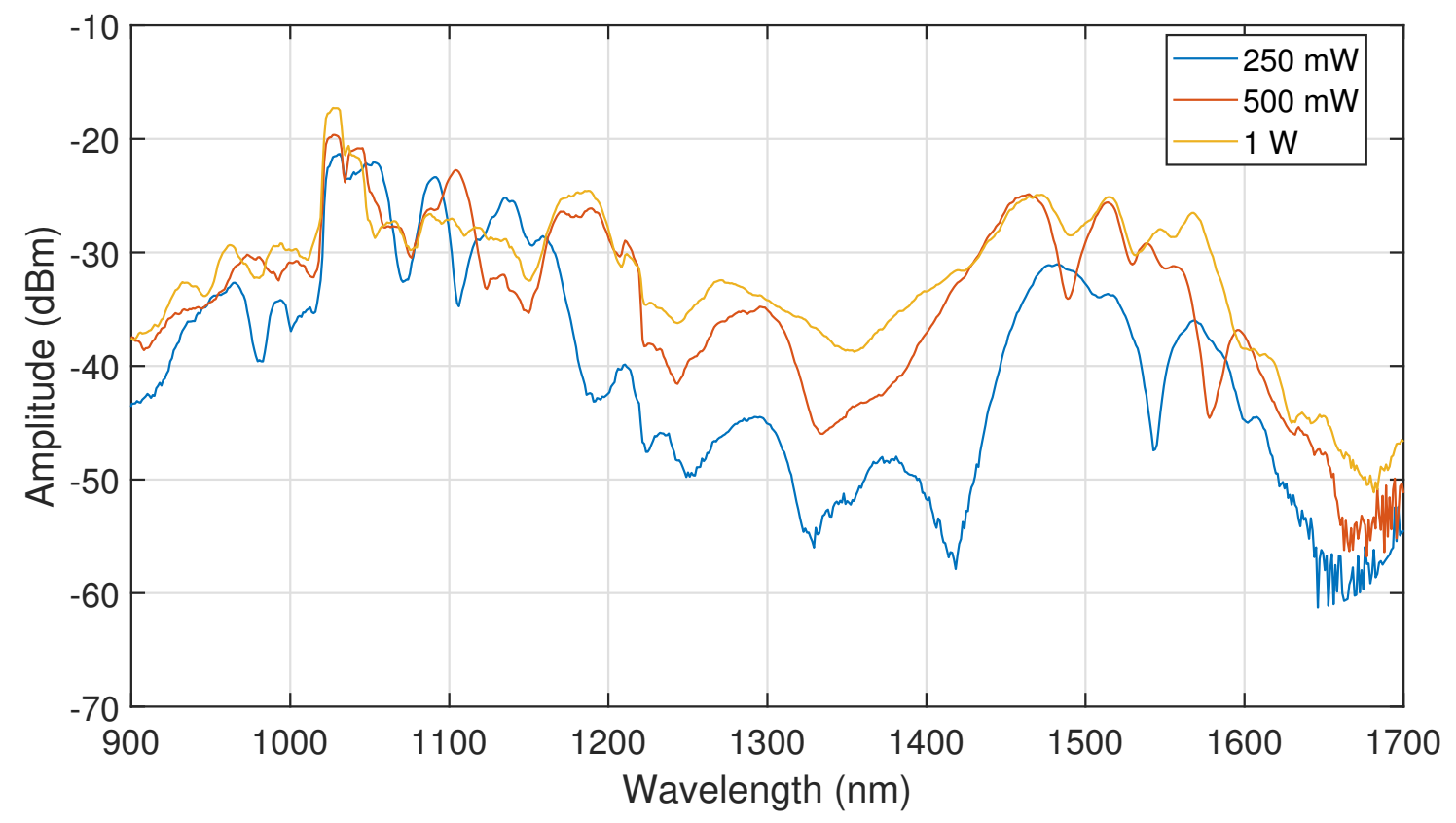

Figure 3. Supercontinuum generated in SiN waveguide with different power levels of $1030 \mathrm{~nm}$ light incident on the coupling lens. The spectrum extends past $1600 \mathrm{~nm}$ even at power levels below $250 \mathrm{~mW}$ with $87 \mathrm{fs}$ pulses at $1030 \mathrm{~nm}$.

The $1560 \mathrm{~nm}$ beam is combined with approximately $6 \mathrm{~W}$ of $1030 \mathrm{~nm}$ light from the Yb amplifier using a dichroic beamsplitter and focused into a $10 \mathrm{~mm} \mathrm{MgO}$ doped periodically poled lithium niobate (PPLN) crystal (Covesion MDFG3-1.0-10, grating period $29.98 \mu \mathrm{m}$ ) designed for difference frequency generation around $3 \mu \mathrm{m}$. A $5 \mathrm{~mm}$ length crystal was also used with similar results, which suggests that the walkoff due to the differing group velocities of the two pulses occurs in $<5 \mathrm{~mm}$. A $100 \mathrm{~mm}$ focal lens AR coated for both wavelengths was used for focusing, and a $100 \mathrm{~mm}$ focal length $\mathrm{CaF}_{2}$ lens AR coated for $3 \mu \mathrm{m}$ wavelength was used to collimate the output. The PPLN crystal was heated to a temperature of $117^{\circ} \mathrm{C}$ for phase matching. After the collimating 
lens an AR coated germanium window was used to separate the pump and signal beams from the MIR idler, with the former two being reflected and the latter being transmitted through the window.

\section{RESULTS}
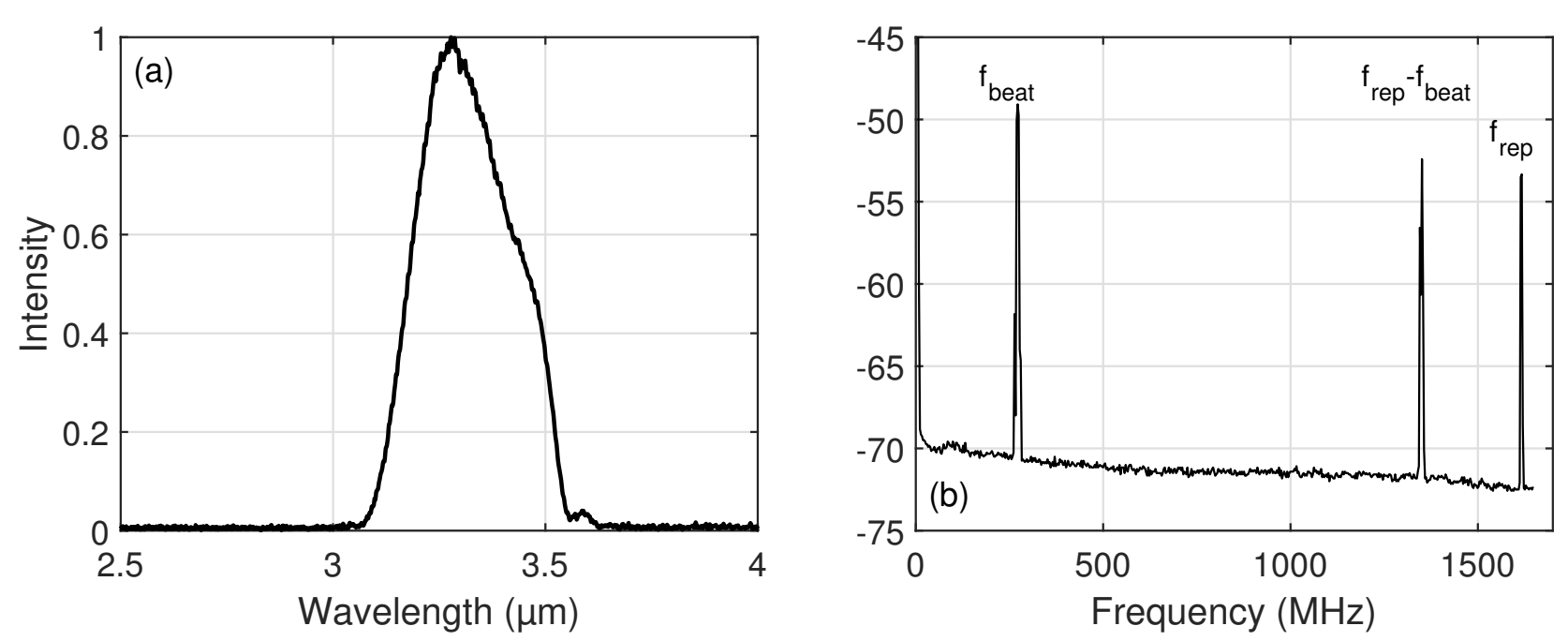

Figure 4. (a) Spectrum of MIR DFG idler output centered at $3.3 \mu \mathrm{m}$. (b) Beat note between doubled MIR comb and $1560 \mathrm{~nm}$ single frequency laser with a $20 \mathrm{~dB}$ SNR. This demonstrates that coherence is preserved in the MIR comb. The $f_{\text {rep }}$ is also visible at $1.61 \mathrm{GHz}$.

In order to verify that coherence was not lost in the spectral broadening in the SiN waveguide or subsequent amplification, a heterodyne beat note measurement was performed between the $1560 \mathrm{~nm}$ output of the amplifier and a single frequency DFB laser operating at $1560.4 \mathrm{~nm}$ (Redfern Integrated Optics). Beat note detection was achieved in free space with an InGaAs photodiode with $1.5 \mathrm{GHz}$ of bandwidth. A beat note was detected with $>25 \mathrm{~dB}$ of SNR, demonstrating that coherence had not been lost while extending the comb from $1030 \mathrm{~nm}$ to $1560 \mathrm{~nm}$. At input powers above $1 \mathrm{~W}$ to the SiN waveguide, the SNR of this beatnote was observed to decrease, possibly indicating a reduction of coherence in the resulting $1560 \mathrm{~nm}$ portion of the comb. Because of this, the input power of $1 \mathrm{~W}$ was chosen to balance the need for enough $1560 \mathrm{~nm}$ power to adequately seed the amplifiers, and the resulting coherence of the broadened comb. MIR output was detected from approximately 3.0-3.5 $\mu \mathrm{m}$ as shown in figure 4 measured with a Fourier transform infrared spectrometer. Maximum power was measured to be $290 \mathrm{~mW}$ using a thermal power meter calibrated for the MIR. Performing the same heterodyne beat measurement with the MIR comb was more difficult because of the difficulty of obtaining a single frequency laser operating in the 3.0-3.5 $\mu \mathrm{m}$ wavelength region. Instead, a second MgO:PPLN crystal was used to frequency double a portion of the MIR comb from $3120 \mathrm{~nm}$ to $1560 \mathrm{~nm}$ to perform a beat note measurement with the same $\mathrm{CW}$ laser as before. In both cases a grating and slits were used to filter out unwanted portions of the spectrum to reduce the noise floor of the beatnote. The detected beat note between the doubled MIR comb and CW laser is shown in 4 with a $20 \mathrm{~dB}$ SNR, demonstrating coherence of the final MIR comb.

\section{SUMMARY}

We developed a frequency comb in the mid-infrared with output from 3.0-3.5 $\mu \mathrm{m}$ with $290 \mathrm{~mW}$ of average power and a repetition rate of $1.61 \mathrm{GHz}$ based on a passively modelocked VECSEL oscillator. The wavelength and repetition rate of this comb source make it uniquely suited to molecular spectroscopy applications. Furthermore, the difference frequency generation that produces the comb ensures that the carrier envelope offset frequency is zero without any active stabilization. This allows for a full comb stabilization by simply locking the repetition rate of the laser. 


\section{ACKNOWLEDGMENTS}

We thank Robert Bedford and Ricky Gibson of AFRL for technical discussions on the experiments. We would like to thank Scott Diddams, Scott Papp, David Carlson, Daniel Hickstein, and Connor Fredrick at NIST for technical discussions and use of the SiN waveguides. We also thank Wolfgang Stolz and Antje Ruiz Perez at Philipps Universitat, Marburg for growing our VECSEL structures, and Ganesh Balakrishnan and Sadhvikas Addamane at the University of New Mexico for growing our SESAMs. This material is based upon work supported by the Air Force Office of Scientific Research under award ID FA9550-17-1-0246.

\section{REFERENCES}

1. G. Wysocki, R. Lewicki, R. Curl, F. Tittel, L. Diehl, F. Capasso, M. Troccoli, G. Hofler, D. Bour, S. Corzine, R. Maulini, M. Giovannini, and J. Faist, "Widely tunable mode-hop free external cavity quantum cascade lasers for high resolution spectroscopy and chemical sensing," Applied Physics B 92, pp. 305-311, Sep 2008.

2. A. Schliesser, N. Picqué, and T. W. Hänsch, "Mid-infrared frequency combs," Nature Photonics 6, pp. 440449, July 2012.

3. H. Guo, C. Herkommer, A. Billat, D. Grassani, C. Zhang, M. H. P. Pfeiffer, W. Weng, C.-S. Brès, and T. J. Kippenberg, "Mid-infrared frequency comb via coherent dispersive wave generation in silicon nitride nanophotonic waveguides," Nature Photonics 12, p. 330, June 2018.

4. C. Erny, K. Moutzouris, J. Biegert, D. Kühlke, F. Adler, A. Leitenstorfer, and U. Keller, "Mid-infrared difference-frequency generation of ultrashort pulses tunable between 3.2 and $4.8 \mu \mathrm{m}$ from a compact fiber source," Opt. Lett. 32, pp. 1138-1140, May 2007.

5. F. Adler, K. C. Cossel, M. J. Thorpe, I. Hartl, M. E. Fermann, and J. Ye, "Phase-stabilized, $1.5 \mathrm{w}$ frequency comb at 2.8-4.8 $\mu \mathrm{m}$," Opt. Lett. 34, pp. 1330-1332, May 2009.

6. A. Baltuška, T. Fuji, and T. Kobayashi, "Controlling the carrier-envelope phase of ultrashort light pulses with optical parametric amplifiers," Phys. Rev. Lett. 88, p. 133901, Mar 2002.

7. H. Timmers, A. Kowligy, A. Lind, F. C. Cruz, N. Nader, M. Silfies, G. Ycas, T. K. Allison, P. G. Schunemann, S. B. Papp, and S. A. Diddams, "Molecular fingerprinting with bright, broadband infrared frequency combs," Optica 5, pp. 727-732, Jun 2018.

8. Y. Deng, F. Lu, and W. H. Knox, "Fiber-laser-based difference frequency generation scheme for carrierenvelope-offset phase stabilization applications," Opt. Express 13, pp. 4589-4593, Jun 2005.

9. F. C. Cruz, D. L. Maser, T. Johnson, G. Ycas, A. Klose, F. R. Giorgetta, I. Coddington, and S. A. Diddams, "Mid-infrared optical frequency combs based on difference frequency generation for molecular spectroscopy," Opt. Express 23, pp. 26814-26824, Oct 2015.

10. C. Hönninger, R. Paschotta, F. Morier-Genoud, M. Moser, and U. Keller, "Q-switching stability limits of continuous-wave passive mode locking," J. Opt. Soc. Am. B 16, pp. 46-56, Jan 1999.

11. A. Klenner and U. Keller, "All-optical q-switching limiter for high-power gigahertz modelocked diodepumped solid-state lasers," Opt. Express 23, pp. 8532-8544, Apr 2015.

12. M. Mangold, C. A. Zaugg, S. M. Link, M. Golling, B. W. Tilma, and U. Keller, "Pulse repetition rate scaling from 5 to $100 \mathrm{ghz}$ with a high-power semiconductor disk laser," Opt. Express 22, pp. 6099-6107, Mar 2014.

13. D. Waldburger, S. M. Link, M. Mangold, C. G. E. Alfieri, E. Gini, M. Golling, B. W. Tilma, and U. Keller, "High-power 100 fs semiconductor disk lasers," Optica 3, pp. 844-852, Aug 2016.

14. A. Laurain, I. Kilen, J. Hader, A. Ruiz Perez, P. Ludewig, W. Stolz, S. Addamane, G. Balakrishnan, S. W. Koch, and J. V. Moloney, "Modeling and experimental realization of modelocked vecsel producing high power sub-100 fs pulses," Applied Physics Letters 113(12), p. 121113, 2018.

15. L. Nugent-Glandorf, T. Neely, F. Adler, A. J. Fleisher, K. C. Cossel, B. Bjork, T. Dinneen, J. Ye, and S. A. Diddams, "Mid-infrared virtually imaged phased array spectrometer for rapid and broadband trace gas detection," Opt. Lett. 37, pp. 3285-3287, Aug 2012.

16. J. M. Dudley, G. Genty, and S. Coen, "Supercontinuum generation in photonic crystal fiber," Rev. Mod. Phys. 78, pp. 1135-1184, Oct 2006. 
17. A. S. Mayer, C. R. Phillips, C. Langrock, A. Klenner, A. R. Johnson, K. Luke, Y. Okawachi, M. Lipson, A. L. Gaeta, M. M. Fejer, and U. Keller, "Offset-free gigahertz midinfrared frequency comb based on optical parametric amplification in a periodically poled lithium niobate waveguide," Phys. Rev. Applied 6, p. 054009, Nov 2016.

18. S. Wang, B. Liu, C. Gu, Y. Song, C. Qian, M. Hu, L. Chai, and C. Wang, "Self-similar evolution in a short fiber amplifier through nonlinear pulse preshaping," Opt. Lett. 38, pp. 296-298, Feb 2013.

19. W. Liu, D. N. Schimpf, T. Eidam, J. Limpert, A. Tünnermann, F. X. Kärtner, and G. Chang, "Pre-chirp managed nonlinear amplification in fibers delivering 100w, 60fs pulses," Opt. Lett. 40, pp. 151-154, Jan 2015.

20. R. Rockmore, C. W. Baker, A. Laurain, T.-H. Wu, R. J. Jones, and J. V. Moloney, "Supercontinuum generation and beatnote detection using ultrafast VECSEL seed oscillators," Proc. SPIE. 10515, 105150M (2018)

21. D. R. Carlson, D. D. Hickstein, A. Lind, S. Droste, D. Westly, N. Nader, I. Coddington, N. R. Newbury, K. Srinivasan, S. A. Diddams, and S. B. Papp, "Self-referenced frequency combs using high-efficiency siliconnitride waveguides," Opt. Lett. 42, pp. 2314-2317, Jun 2017. 